\title{
Metalloproteinases and their inhibitors as therapeutic targets for multiple sclerosis: current evidence and future perspectives
}

This article was published in the following Dove Press journal:

Metalloproteinases In Medicine

20 January 2017

Number of times this article has been viewed

\author{
Tiziana Latronico \\ Grazia Maria Liuzzi \\ Department of Biosciences, \\ Biotechnologies, and \\ Biopharmaceutics, Aldo Moro \\ University of Bari, Bari, Italy
}

Correspondence: Grazia Maria Liuzzi Department of Biosciences, Biotechnologies, and Biopharmaceutics, Aldo Moro University of Bari, 4 Via Orabona, Bari 70125, Italy Email graziamaria.liuzzi@uniba.it

\begin{abstract}
The treatment of multiple sclerosis (MS) has seen important changes in the last two decades with the introduction of several drugs able to modify the evolution of this disease. Current MS therapies primarily target the peripheral immune response, although it has been suggested that their efficacy could be in part the result of the beneficial effect on other nonspecific targets, such as matrix metalloproteinases (MMPs). Numerous experimental studies have suggested that MMPs may be involved in MS pathogenesis by contributing to blood-brain barrier disruption, migration of leukocytes into the central nervous system, demyelination and axonal damage. Therefore, MMPs have been considered important therapeutic targets in the course of MS. In this respect, different attempts have been made to develop synthetic, low-molecular-weight inhibitors of MMPs for the potential treatment of diseases in which MMPs play a major role. However, technical difficulties, side effects and reduced patient compliance because of parenteral administration have greatly limited the development in the clinical practice of specific anti-MMP drugs. By contrast, interesting results have been obtained with compounds that are already used in the clinical practice, such as MS drugs and natural compounds with anti-inflammatory and antioxidant activity. Here, we discuss the evidence and potential mechanisms for altered MMP function in MS. Furthermore, we outline the possible medical implications for the use of compounds that target MMP activity and we propose that together with anti-MS drugs, other compounds with anti-inflammatory and antioxidant properties, such as natural $\omega 3$ fatty acids, polyphenols and tetracyclines, which inhibit MMP functions, might represent potential therapeutic approaches to mitigate MMP-related damage during MS.
\end{abstract}

Keywords: multiple sclerosis, matrix metalloproteinases, therapy, matrix metalloproteinase inhibitors (MMPI), central nervous system, neuroinflammation

\section{Introduction}

Multiple sclerosis (MS) is a chronic inflammatory disease of the central nervous system (CNS) that represents a leading cause of neurological disability among young adults. Hallmarks of MS are blood-brain barrier (BBB) disruption, migration of leukocytes into the CNS, demyelination and axonal and oligodendrocyte damage. ${ }^{1}$ Much experimental evidence indicates that MMPs are actively involved at different levels in MS pathogenesis. ${ }^{2}$ Therefore, many attempts have been made to downregulate MMP activity and expression in order to limit tissue damage occurring in MS (Table 1). The current management of MS patients is challenging and includes pharmacotherapy with corticosteroids, antibodies (Abs) and immunomodulant and immunosuppressive $\operatorname{drugs}^{3}$ (Box 1). All the therapies currently used can only slow disease progression 
Table I MMP inhibitors in MS clinical development*

\begin{tabular}{|c|c|c|c|c|c|c|c|}
\hline Type & Class & Agent & Specificity & $\begin{array}{l}\text { Experimental } \\
\text { model }\end{array}$ & $\begin{array}{l}\text { Sample } \\
\text { analyzed }\end{array}$ & Remarks & Reference \\
\hline \multirow[t]{3}{*}{$\begin{array}{l}\text { Synthetic MMP } \\
\text { inhibitors }\end{array}$} & $\begin{array}{l}\text { Hydroxamate MMP } \\
\text { inhibitors }\end{array}$ & $\mathrm{BBIOI}$ & MMP-7, MMP-26 & $\begin{array}{l}\text { Acute EAE in } \\
\text { Lewis rats }\end{array}$ & $\begin{array}{l}\text { Spinal } \\
\text { cord, CSF }\end{array}$ & & 79 \\
\hline & & Ro3I-9790 & Broad spectrum & $\begin{array}{l}\text { Acute EAE in } \\
\text { Lewis rats }\end{array}$ & CNS tissue & & 81 \\
\hline & & GM600I & MMP-2, MMP-9 & $\begin{array}{l}\mathrm{EAE} \text { in } \mathrm{SJL} / \mathrm{J} \\
\text { mice }\end{array}$ & CSF & & 82 \\
\hline \multirow{4}{*}{$\begin{array}{l}\text { Anti- } \\
\text { inflammatory } \\
\text { drugs }\end{array}$} & $\begin{array}{l}\text { Modified } \\
\text { tetracyclines }\end{array}$ & Minocycline & MMP-9 & $\begin{array}{l}\mathrm{EAE} \text { in C57BL/6 } \\
\text { mice }\end{array}$ & Spinal cord & & 93 \\
\hline & & & MMP-2 & $\mathrm{EAE}$ in DA rats & CNS tissue & & 94 \\
\hline & & & MMP-9 & $\begin{array}{l}\text { Pilot study in } \\
\text { RR-MS patients }\end{array}$ & Serum & & 96 \\
\hline & Antibiotics & Doxycycline & MMP-9 & $\begin{array}{l}\text { Open-label } \\
\text { trial in RR-MS } \\
\text { patients }\end{array}$ & Serum & $\begin{array}{l}\text { Used in } \\
\text { combination } \\
\text { with IFN } \beta\end{array}$ & 97 \\
\hline \multirow[t]{7}{*}{ Anti-MS drugs } & Corticosteroids & Methylprednisolone & MMP-9 & MS patients & CSF & & 61 \\
\hline & $\begin{array}{l}\text { Immunomodulant } \\
\text { drugs }\end{array}$ & IFN $\beta$ & MMP-9 & RR-MS patients & Serum & & 6 \\
\hline & & & MMP-9 & RR-MS patients & Serum & & 87 \\
\hline & & & MMP-2, MMP-9 & RR-MS patients & $\begin{array}{l}\text { CSF, } \\
\text { serum }\end{array}$ & & 88 \\
\hline & $\begin{array}{l}\text { Immunosuppressive } \\
\text { drugs }\end{array}$ & Mitoxantrone & MMP-9 & $\begin{array}{l}\text { RR-and SP-MS } \\
\text { patients }\end{array}$ & PBMC & & 7 \\
\hline & & Fingolimod (FTY720) & MMP-9 & $E A E$ in DA rats & Spinal cord & & 8 \\
\hline & Antibodies & Natalizumab & MMP-9 & $\begin{array}{l}\text { Open-label } \\
\text { trial in RR-MS } \\
\text { patients }\end{array}$ & CSF & & 9 \\
\hline \multirow[t]{5}{*}{$\begin{array}{l}\text { Compounds from } \\
\text { natural sources }\end{array}$} & Fatty acids & $\omega 3$ PUFA & MMP-9 & $\begin{array}{l}\text { Open-label } \\
\text { trial in RR-MS } \\
\text { patients }\end{array}$ & $\begin{array}{l}\text { PBMC, } \\
\text { serum }\end{array}$ & & 49 \\
\hline & Flavonoids & Quercetin & MMP-9 & RR-MS patients & PBMC & $\begin{array}{l}\text { Used in } \\
\text { vitro in } \\
\text { combination } \\
\text { with IFN } \beta\end{array}$ & 41 \\
\hline & Thiol compounds & $\alpha$-Lipoic acid & MMP-9 & EAE in SJL mice & & & 101 \\
\hline & & & MMP-9 & MS patients & Serum & & 102 \\
\hline & & $\begin{array}{l}\text { Combination } \\
\text { of antioxidants } \\
\text { ( } \omega 3, \alpha \text {-lipoic } \\
\text { acid, resveratrol), } \\
\text { multivitamin } \\
\text { complex, and } \\
\text { vitamin D }\end{array}$ & MMP-9 & $\begin{array}{l}\text { Pilot study in } \\
\text { RR- and PP-MS } \\
\text { patients }\end{array}$ & Serum & $\begin{array}{l}\text { Used in } \\
\text { combination } \\
\text { with IFN } \beta\end{array}$ & 12 \\
\hline
\end{tabular}

Note: *The list of MMP inhibitors is not exhaustive; it refers to drugs and compounds used for the treatment of MS in clinical trials and in human and animal studies and to those cited in the paper.

Abbreviations: PUFA, polyunsaturated fatty acid; PBMC, peripheral blood mononuclear cells; CSF, cerebrospinal fluid; DA, dark agouti; EAE, experimental allergic encephalomyelitis; RR-MS, relapsing-remitting multiple sclerosis; PP-MS, primary progressive MS; SP-MS, secondary progressive MS; SJL, Swiss/Jackson Laboratory.

and prevent some disability symptoms, and this is perhaps one of the reasons why the scientific community is geared to support conventional treatments with the use of generic drugs or natural compounds with anti-inflammatory and antioxidant action, combined with a specific diet. ${ }^{4,5}$ Several studies have shown that both anti-MS-specific therapies ${ }^{6-9}$ and some antioxidant and anti-inflammatory compounds have the ability to downregulate MMPs, suggesting that inhibition of MMPs may represent an additional mechanism by which these agents decrease the development of new CNS lesions in the course of MS. ${ }^{10-12}$

In this review, we summarize the general characteristics of the different MMPs and their regulatory mechanisms. The pathogenic role of MMPs in the development and progression 


\section{Box I}

Treatment in multiple sclerosis

Numerous challenges affect the medical community for the treatment of multiple sclerosis (MS), since there are no drugs able to cure this disease. MS treatment typically focuses on slowing the progression of the disease and minimizing MS-related disability. The choice of therapy will depend on the phase and clinical activity of the disease. Generally, therapies aim at regulating the immune system with different mechanisms: inhibition of $T$ and $B$ lymphocytes, monocyte and macrophage replication, or inhibition of cell migration by using immunomodulatory and immunosuppressive agents or antibodies against the antigens expressed on the cell surface.

Currently, the drugs approved by the US Food and Drug Administration and the European Medicines Agency consist of corticosteroids, such as methylprednisolone, immunomodulatory drugs, such as IFN $\beta$ - Ia or - Ib, glatiramer acetate and dimethyl fumarate, immunosuppressive drugs, such as mitoxantrone and fingolimod, or monoclonal antibodies, such as natalizumab and alemtuzumab. Other therapies, not officially approved, consist in the use of azathioprine and cyclophosphamide, two immunosuppressive drugs, and methotrexate, an antimetabolite that limits cellular replication by acting as an antagonist of folic acid. Ocrelizumab, a second-generation humanized anti-CD20 antibody, was explored in a large Phase II trial in patients with relapsing-remitting MS, as well as in primary progressive MS. In a multicenter, randomized, Phase III study, patients with progressive MS treated with ocrelizumab showed less disability than placebos. ${ }^{103}$ These results indicate that ocrelizumab is a promising drug for the treatment of the progressive forms of MS for which the most used therapies are ineffective.

The complexity and heterogeneity of MS pathogenesis makes for often-ineffective therapies. Therefore, a combination of drugs that act by different mechanisms of action may be a therapeutic strategy that is more effective. Indeed, unlike a single drug, the use of combination therapy would target the different mechanisms and pathways that occur concomitantly in MS pathogenesis. Combination therapy has thus become attractive, since it aims not only to reduce inflammatory processes but also to preserve neuroprotective functions. In this respect, in vitro experiments, animal studies, and clinical trials have achieved favorable results. ${ }^{104,105}$

Currently, many people with MS explore complementary and alternative medicine treatments. In this respect, a low-fat diet and supplementation of pharmacological therapy with antioxidant and anti-inflammatory natural compounds has proved to be highly promising in the treatment of MS patients. ${ }^{12}$

of MS is also highlighted. Finally, we discuss the different therapeutic approaches to target MMPs selectively and their potential effects in the treatment of MS.

\section{Biology of MMPs Structure of MMPs}

MMPs are a large family of neutral, $\mathrm{Zn}^{2+}$-dependent endopeptidases belonging to the metzincins that have as major targets components of the extracellular matrix (ECM), such as collagen, elastin, fibronectin and laminin. MMPs from vertebrates are classified according to their sequence similarity and substrate specificity in collagenases, stromelysins, gelatinases, matrilysins, membrane-type MMPs (MT-MMPs) and other MMPs with no group designation.

MMPs have a common basic structure consisting of a predomain, a propeptide, a catalytic domain with a highly conserved zinc-binding site, a hinge region, a hemopexin-like domain and a transmembrane domain. ${ }^{13}$ The pre-domain is an $\mathrm{N}$-terminal amine hydrophobic signaling sequence that directs, after synthesis, MMPs to the endoplasmic reticulum. The propeptide contains a cysteine residue within a conserved inhibitory sequence, which binds zinc in the active site of the catalytic domain maintaining the enzyme in a latent or an inactive state (zymogen) until the propeptide is proteolytically cleaved. The C-terminal hemopexin-like domain is important for the recognition of the substrate and of their natural tissue inhibitors (TIMPs). This domain is connected to the catalytic domain by a hinge region, represented by a proline-rich peptide sequence that varies in length and influences substrate specificity.

Although MMPs share some structural characteristics, MMPs can also be distinguished based on the absence or presence of domains. For this reason, MMPs have been further classified into the major subgroups of gelatinases, archetypal MMPs, matrilysins and furin-activated MMPs (Figure 1). Gelatinases contain three repeats of fibronectin type II inserts in the catalytic domain, which facilitate binding to gelatin and collagen. The matrilysins, the smallest MMPs, possess a basic structure lacking the hemopexin-like domain. The archetypal MMPs are characterized by a propeptide, a catalytic domain, and a hemopexin domain. The furin-activated MMPs contain a furin-recognition motif inserted between the propeptide and the catalytic domain, which are cleaved by convertases, allowing intracellular activation. The MT-MMPs include type-I transmembrane MT-MMPs and glycosylphosphatidylinositol MT-MMPs. The glycosylphosphatidylinositol MT-MMPs contain membrane-anchoring domains, whereas the type I transmembrane MT-MMPs have a carboxy-terminal transmembrane domain and a cytoplasmic domain.

\section{Regulation of MMP expression and activity}

MMPs play important roles in both physiological and pathological processes, including turnover and remodeling of the ECM, embryogenesis, cell migration, angiogenesis, invasion of metastatic cells, neuroinflammation and other harmful events. Therefore, MMPs are tightly regulated at various levels: gene transcription, post-transcription, activation of zymogens by removal of the prodomain and inhibition by the specific endogenous inhibitors TIMPs and by nonspecific proteinase inhibitors, such as $\alpha_{2}$-macroglobulin. 


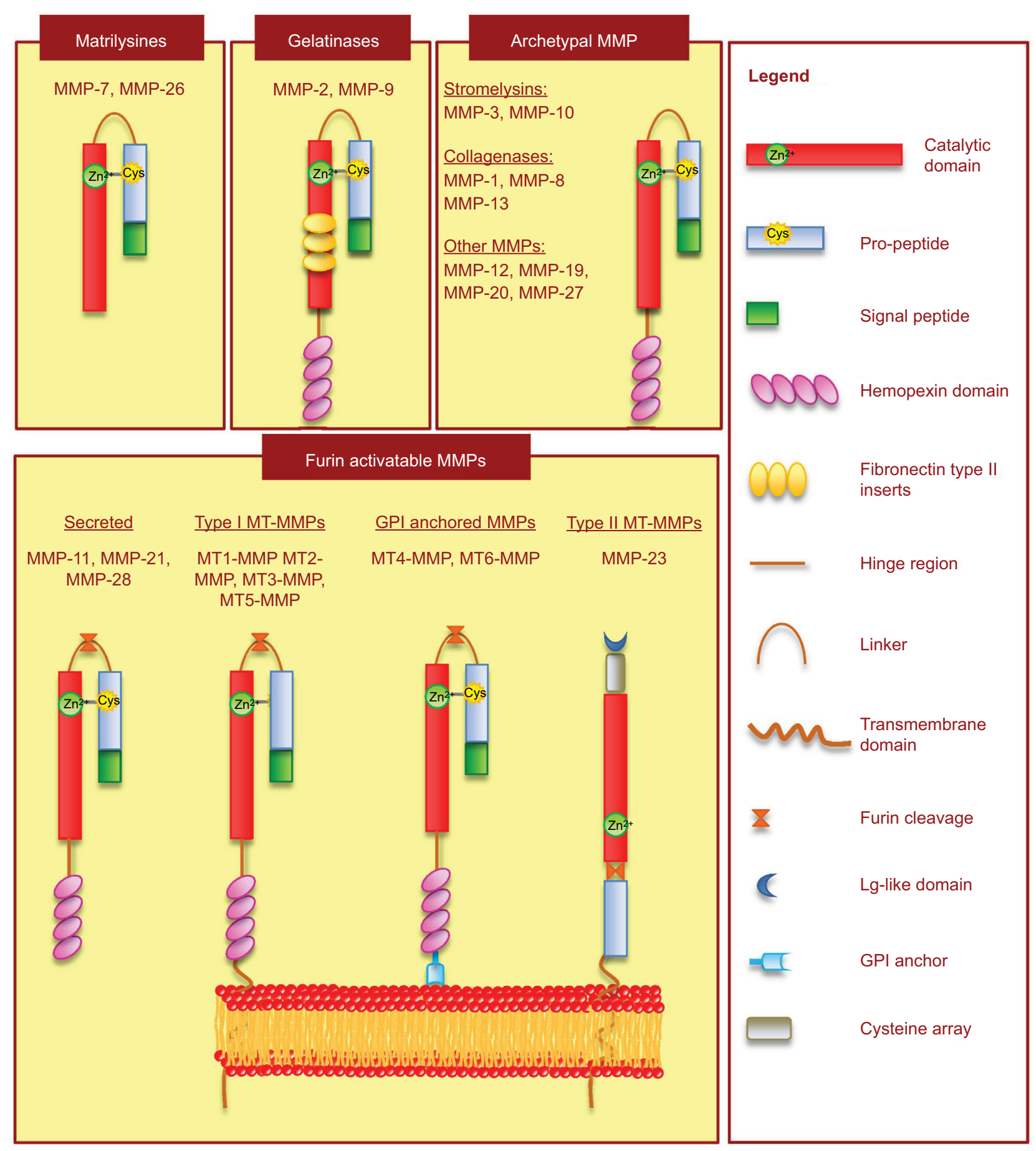

Figure I Schematic representation of MMP structure based on domain arrangement. Abbreviations: MT-MMP, membrane-type MMP; GPI, glycosylphosphatidylinositol.

Many MMPs are constitutively expressed in some cell types, but their levels may be induced in response to endogenous or exogenous stimuli, such as growth factors, hormones, steroids, cytokines, chemokines, pathogens and cell-cell or cell-ECM interactions. Most of the transcriptional regulation mechanisms, mediated by soluble factors, occur mainly following the binding of the stimulating factors to their cell-surface receptors. These extracellular stimuli induce the activation of transcription factors through phosphorylation processes regulated by the activation of a series of signal-transduction factors. In particular, the transcriptional regulation of many MMP genes is mediated mainly through activation of the transcription factors NFKB and AP1, via the JNK-SAPK, ERK1-2 or p38 MAPK pathways. ${ }^{14}$ 
The activation of latent enzymes is an important critical point of post-transcriptional regulation. MMPs are synthesized as inactive forms and activated following secretion by a "cysteine switch" mechanism consisting in the cleavage of the hydrophobic propeptide domain. This is a process that may be mediated by proteases, including other MMPs, such as MT-MMPs, or chemically by organomercurial substances, urea, some detergents, reactive oxygen species (ROS) and hormones. In vivo, an important activator of MMPs is represented by the plasmin/plasminogen-activator system. ${ }^{15}$

\section{Endogenous inhibitors of MMPs}

MMP activity is regulated by two main endogenous inhibitors: $\alpha_{2}$-macroglobulin and TIMPs. $\alpha_{2}$-Macroglobulin is a plasmatic glycoprotein that is not a specific inhibitor for MMPs, but in general has a regulatory function toward a wide spectrum of endoproteinases; ${ }^{16}$ it regulates MMPs in the extracellular environment through their binding and subsequent removal through endocytosis. ${ }^{17}$ TIMPs are the major and more specific inhibitors of MMPs. They act by inactivating the enzyme through a 1:1 noncovalent bond. TIMPs are dimers variably glycosylated with a smaller $\mathrm{C}$ domain and a highly conserved $\mathrm{N}$-terminal region that contains a sequence able to bind the catalytic domain of MMPs and that is responsible for MMP inhibition. The C-terminal domain of TIMPs stabilizes their binding to pro-MMPs by interactions with the hemopexin domain. Four different TIMPs (TIMP-1, -2, -3, and -4) have been identified and characterized in mammals. TIMP-1, -2, and -4 are secreted proteins, while TIMP-3 is associated with membrane-bound proteins in both its glycosylated and unglycosylated form. TIMPs share structural features but exhibit different biochemical properties and expression patterns. ${ }^{16}$ All TIMPs are broad-spectrum inhibitors of MMPs, but show differences in their specificity. TIMP-1 mainly inhibits MMP-9, whereas TIMP-2 inhibits MMP-2. Among TIMPs, TIMP-2 and TIMP-3 are effective inhibitors of MT-MMPs, ${ }^{18}$ while only TIMP-3 is able to inhibit TACE. ${ }^{19}$

The balance between MMP and TIMP is important in maintaining tissue homeostasis, and indeed an imbalance between TIMPs and MMPs, due to the dysregulation of TIMP or MMP expression, might lead to excessive degradation of matrix or to matrix apposition and thus contribute to the pathogenesis of different diseases.

\section{Exogenous synthetic MMP inhibitors}

The involvement of MMPs in numerous diseases, such as tumor-cell invasion, autoimmunity and neuroinflammation, has led the scientific community to identify these enzymes as possible therapeutic targets in these pathologies and to search and synthesize molecules with inhibitory activity against MMPs. In general, the early MMP inhibitors (MMPIs) were small molecules mimicking the structure of the natural peptide substrates, the "peptidomimetic" MMPIs. Afterward, MMPIs, possessing a zinc-binding group ( $\mathrm{ZBG}$ ) able to inactivate the enzyme by chelating the catalytic $\mathrm{Zn}^{2+}$ ion in the active site, were synthesized. ${ }^{20}$ The ZBG included hydroxamic acids, carboxylates, thiols and phosphonic acids. The most potent MMPIs are those with hydroxamic acid ZBG, with a broad spectrum of inhibition, a strong MMP-inhibitory activity, thus able to inhibit MMPs at low concentrations. Despite being potent inhibitors, they have not been approved by the US Food and Drug Administration (FDA) for clinical trials, as many of them, such as batimastat, an MMPI proposed for cancer treatment, have shown poor oral bioavailability and adverse musculoskeletal side effects, ${ }^{21}$ probably due to their poor specificity and selectivity. Therefore, despite structural knowledge of the catalytic domain of various MMPs, the development of highly specific synthetic MMPIs able to discriminate among the different members of MMP family remains a challenge. Another challenge in the therapeutic use of synthetic MMPIs comes from experimental evidence that MMPs may have protective functions in the course of a disease, such as in repression of tumor angiogenesis and inactivation of chemokines involved in the metastatic process $^{22}$ or in remyelination in the course of neuroinflammatory diseases. ${ }^{23}$ Therefore, nonspecific MMP blocking would be harmful for the organism rather than beneficial. For these reasons, the research has turned its interest toward the development of inhibitors with selectivity for a single MMP, such as the ZBG-lacking MMPIs. ${ }^{20}$ In fact, the catalytic $\mathrm{Zn}$ site represents the most preserved site among those of MMPs, and thus inhibitors that bind other sites would result in being more selective toward the MMP target. This is the case of the selective MMPIs against MMP-13, which have shown encouraging results in preclinical studies of osteoarthritis models. ${ }^{24}$ Another promising class of ZBG-lacking MMPIs is represented by the "suicide inhibitors", also known as "mechanism-based inhibitors". These MMPIs contain a specific group that coordinate the $\mathrm{Zn}^{2+}$ in the active site, resulting in a conformational change and a covalent attachment that prevent MMP dissociation. ${ }^{25}$ The first mechanism-based inhibitor of MMPs was SB3CT, a selective inhibitor toward MMP-2 and MMP-9, that, in preclinical studies, has shown anticancer properties, ${ }^{26}$ as well 
as the ability to prevent damage during cerebral ischemia ${ }^{27}$ and to attenuate damage resulting from traumatic brain injury. ${ }^{28}$ An attempt to develop specific inhibitors of MMP-2 and MMP-9 was made by synthesizing cyclic peptides corresponding to the frequently observed motifs in MMPactive sites, thus preventing substrate access. Two cyclic peptides - CRRHWGFEFC and CTTHWGFTLC - were found to be selective inhibitors of MMP-9 and MMP-2. ${ }^{29}$ Nevertheless, both peptides are quickly metabolized, and thus they are poorly suitable for therapeutic purposes.

In addition to synthetic compounds, antibiotics have also been frequently used as suitable MMPIs. In addition to their antibiotic action, chemically modified tetracyclines (CMTs), such as minocycline and doxycycline, have shown anti-inflammatory effects, such as downregulation of MMPs, and proinflammatory factors, together with the ability to cross the BBB. ${ }^{30}$ Unlike conventional tetracyclines, semisynthetic tetracyclines have been shown to possess high therapeutic potential in the neurological field for their immunomodulatory and neuroprotective effects. ${ }^{31,32}$ Experimental evidence has reported that among CMTs, minocycline is the most potent MMPI. CMTs inhibit MMPs via different mechanisms: they can directly inhibit enzyme activity or indirectly affect MMP gene expression. ${ }^{33}$

Another innovative approach for MMP inhibition is represented by $\mathrm{Abs}$. In this respect, a monoclonal $\mathrm{Ab}$ (REGA3G12) selective for MMP-9 ${ }^{34}$ and an Ab (DX2400) $)^{35}$ able to inhibit MT1-MMP were developed. The use of Abs is promising, since they are able to target the specific MMP selectively, but their rapid proteolysis and removal from circulation have limited their use. Other new attempts are aimed at inhibiting the transcription factors involved in MMP-gene expression or in directly silencing MMP genes. RNA interference appears to have great potential as a novel therapeutic strategy, based on the highly specific and efficient silencing of a target gene. Recently, nanoparticles (NPs) have been used as a nonviral vector to deliver MMP-9 small interfering RNA (siRNA) in brain microvascular endothelial cells, proving to be a good method to maintain the integrity of the BBB. ${ }^{36,37}$

\section{Exogenous natural MMP inhibitors}

Terrestrial and marine organisms represent a rich source of bioactive compounds with anti-inflammatory, antiangiogenic, anticancer and antioxidant properties. Among them, polyphenols, polyunsaturated fatty acids (PUFAs) and other natural compounds have shown high pharmacologic and therapeutic potential. Experimental evidence has suggested that the anti-inflammatory actions of several bioactive compounds are associated with their abilities to inhibit the expression and activity of MMPs. In this respect, numerous studies have indicated that polyphenolic compounds, such as resveratrol, quercetin, catechins, epigallocatechin, hydroxytyrosol, and others, are able to inhibit MMPs via different mechanisms, including the blocking of enzymatic activity and the reduction of gene expression. ${ }^{11,38-41}$

The effect of polyphenols on the regulation of MMPs may be the indirect result of the cellular redox-restoration state through ROS scavenging. Indeed, emerging findings suggest that ROS may play a role in enhancing MMP-9 through the activation of the MAPK pathway and transcription factors, such as NFKB and AP1. ${ }^{42}$ Therefore, by reducing ROS levels, polyphenols counteract MMP-9 production.

In addition, polyphenols can directly inhibit MMP levels through the activation of enzymes, such as AMPK and sirtuins (Sirt), and nuclear receptors, such as the peroxisome proliferator-activated receptors PPAR $\alpha,-\beta$, and $-\delta / \lambda$, or by the inhibition of the transcription factors NFKB, AP1 and mTOR, all implicated in phenomena that regulate cell survival, aging, apoptosis and stress resistance. In particular, the different isotypes of the PPARs downregulate the activity of NFKB and AP1 and the transcription of various molecules involved in inflammation and autoimmunity. ${ }^{43}$

Clinical and experimental studies in animal and in vitro models have indicated that $\omega 3$ PUFAs also have important bioactive properties with anti-inflammatory and immunomodulant abilities. ${ }^{4-46} \omega 3$ PUFAs have shown beneficial effects in different pathologies, such as cardiovascular, neurological and inflammatory diseases. ${ }^{47,48}$ In this respect, it has been reported that $\omega 3$ FAs are able to decrease the production of MMPs. In particular, it has been demonstrated that $\omega 3$ PUFAs inhibited in vitro MMP-9 production in lipopolysaccharide-activated microglia. ${ }^{10}$ The mechanisms by which $\omega 3$ PUFAs downregulate the production of MMPs may be related to their inhibitory effect on proinflammatory cytokines involved in the induction of MMPs, such as TNF $\alpha$, IL-1 and IL-2. ${ }^{49}$ However, it has been suggested that $\omega 3$ PUFAs may directly inhibit MMP gene transcription through their inhibitory effect on NFKB- and AP1-binding activity. ${ }^{50}$ Other compounds, such as saccharoids, flavonoids, polyphones and FAs extracted from marine organisms, have the ability to inhibit MMP activity, indicating high pharmacological potential in different inflammatory diseases. ${ }^{51,52}$ Recently, it has been demonstrated that aqueous extracts from marine sponges inhibit the activity and expression 
of MMP-2 and MMP-9 in an in vitro model or rat primary astrocytes. $^{53}$

\section{Matrix metalloproteinases: link to multiple sclerosis}

MS is a complex, chronic, autoimmune disease of the CNS that affects approximately 400,000 young adults worldwide. $^{54}$ The inflammation is responsible of the formation of plaques that are predominantly located in the CNS white matter. People with MS show a partial or complete loss of the functions controlled by the CNS. The symptoms and signs of MS can reflect the anatomical sites and the intensity of their damage. Three main forms of MS, with different pathological findings, can be recognized. Approximately $85 \%$ of patients manifest a relapsing-remitting form (RR-MS) characterized by the appearance of neurological symptoms (relapsing phase), followed by a complete or partial recovery of lost functions during the remitting phase. About $50 \%$ of patients initially diagnosed with RR-MS develop the secondary progressive (SP-MS) form characterized by a rapid progression of clinical and neurological damage. SP-MS is characterized by less inflammatory lesions compared to the RR-MS, but the severity of the disease continues to progress, probably due to the increased axonal loss. Some patients may experience a primary progressive form (PP-MS) at the onset of the disease. This form is characterized by a gradual progression of the disease, resulting in a decline in physical abilities of the patient.

MS is a complex mutifactorial disease with unknown etiology, in which both viral and nonviral environmental factors and genetic traits determine the susceptibility to develop the disease. ${ }^{55,56}$ The link between genetic susceptibility and environmental factors in the development of MS is probably represented by human endogenous retroviruses. ${ }^{57}$

MS pathogenesis is only partially understood. Experimental studies from animal models suggest that MS is an autoimmune, inflammatory disease characterized by BBB disruption, perivascular inflammation, axonal injury and breakdown of the myelin sheath. ${ }^{1}$ The extravasation of autoreactive $\mathrm{CD}^{+} \mathrm{T}$ cells, directed toward myelin, together with activated macrophages and microglia, release toxic inflammatory mediators that are responsible for myelin degradation. ${ }^{58}$ In parallel with inflammatory demyelination, axonal damage occurs in the early phase, and probably represents a main cause of later permanent disability. ${ }^{59}$ AutoAbs crossing the BBB or locally produced by B cells and complement activation also contribute to myelin degradation and axonal loss. ${ }^{60}$
Several studies have suggested that MMPs are key mediators in the pathogenesis of MS. Mechanisms by which MMP contribute to MS pathology include disruption of the BBB and transmigration of inflammatory cells into the $\mathrm{CNS},{ }^{61,62}$ proteolytic cleavage of membrane-bound proinflammatory cytokines ${ }^{63}$ and degradation of the myelin sheath and axonal damage ${ }^{64,65}$ (Figure 2). Elevated expression of different MMPs, such as MMP-1, MMP-2, MMP-3, MMP-7, and MMP-12, has been found in CNS lesions and in blood monocytes from MS patients. ${ }^{66-69}$ Other authors have found increased levels of monocyte-derived MMP-2 and MMP-14 transcripts in samples from MS patients in relation to the progression of the disease..$^{70}$ Among the MMPs, MMP-9 has been the most extensively studied, since it displays the most important alterations in the course of MS. Several reports have demonstrated the increase of MMP-9 in the brain tissue, cerebrospinal fluid (CSF) and blood circulation of patients with RR-MS. ${ }^{71-74}$ Serum levels of MMP-9/TIMP-1 and MMP-9 increase in RR-MS patients during acute exacerbations and correlate with the degree of BBB disruption, as evidenced by the number of enhancing magnetic resonance imaging (MRI) lesions. ${ }^{61,74,75}$ By contrast, both serum and CSF levels of TIMP-1 decrease, suggesting that an imbalance between MMP and TIMP is a crucial pathogenetic feature in MS. ${ }^{73}$ As reported by Liuzzi et al, ${ }^{73}$ the increase in MMP-9 in the CSF of MS patients may reflect not only release from transmigrated leukocytes but also its intrathecal production. The synthesis of MMP-9 within the CNS may reflect the release of this enzyme from resident CNS cells, as well as from activated macrophages present in active lesions, suggesting a role for MMP-9 not only in lymphocyte trafficking across the BBB but also in demyelination.

Since MMP-9 is able to degrade MBP, the release of this enzyme in the brain parenchyma in the proximity of the myelin sheath could directly contribute to its degradation and to the release into the CNS of encephalitogenic fragments of MBP or other myelin antigens. ${ }^{76,77}$ In addition, an indirect contribution of MMP-9 to demyelination could also come from its ability to release the membrane-bound form of the proinflammatory $\mathrm{TNF} \alpha{ }^{78}$

\section{Pharmacological blocking of MMPs in multiple sclerosis}

The involvement of MMPs in the inflammatory demyelination and neurodegenerative processes occurring in MS has increasingly convinced the scientific community that these enzymes represent a good therapeutic target. In the nineties, the synthetic MMPIs, which have been extensively tested for 

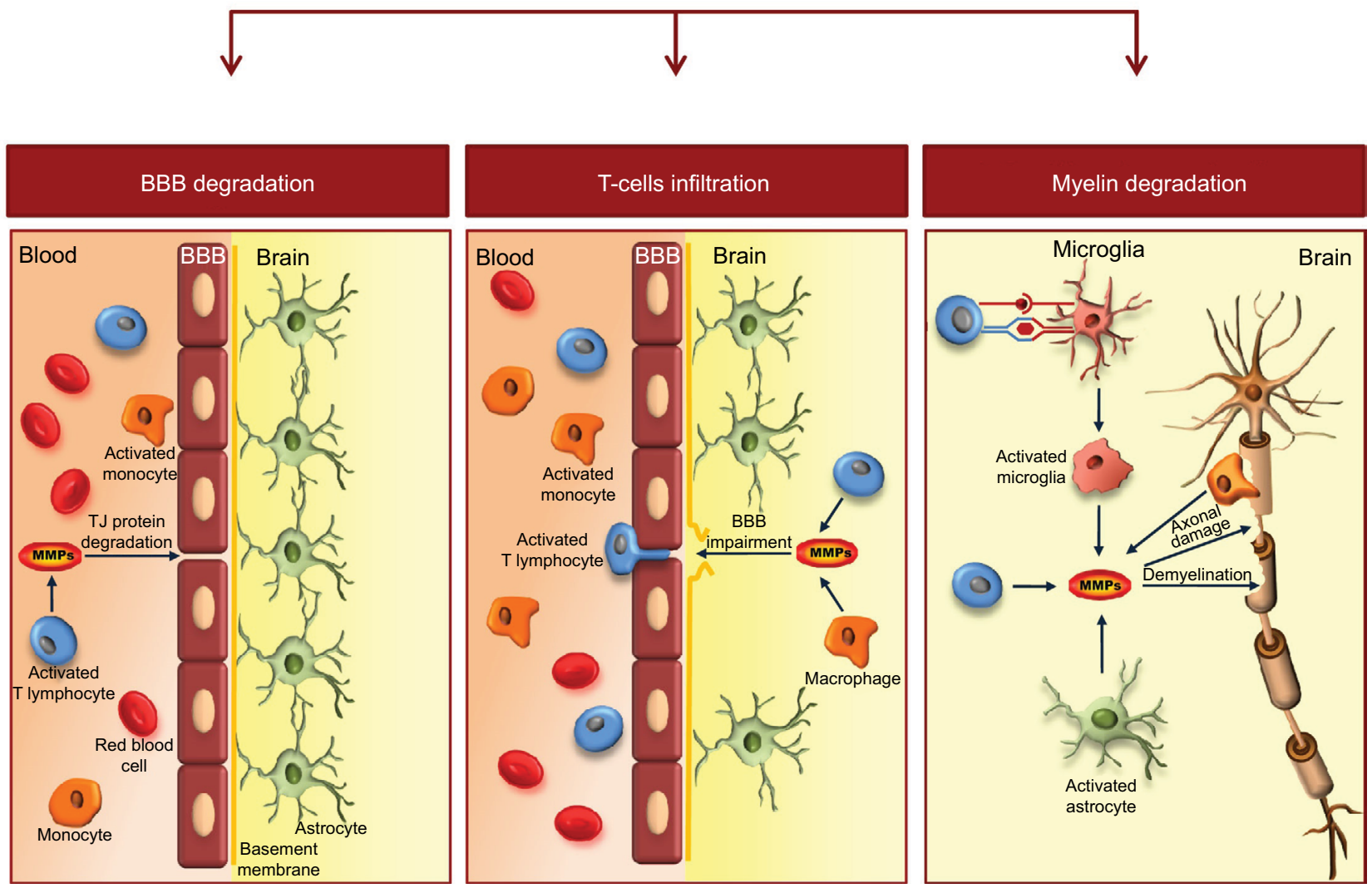

Figure 2 Schematic representation of the involvement of MMPs in MS pathology.

Notes: MMPs, and in particular MMP-9, are involved in the mechanisms of BBB disruption through degradation of TJ proteins. This allows extravasation of autoreactive $\mathrm{CD}^{+} \mathrm{T}$ cells into the CNS. Within the brain parenchyma, activated T lymphocytes release inflammatory cytokines and MMPs. Moreover, T cells activate microglia cells/ macrophages and astrocytes to the release of proinflammatory cytokines and MMPs, which contribute to myelin degradation and axonal loss.

Abbreviations: MS, multiple sclerosis; BBB, blood-brain barrier.

cancer therapy, became attractive for their therapeutic use in neurological diseases. Studies on animals with experimental allergic encephalomyelitis (EAE) treated with hydroxamate MMPIs such as BB101, ${ }^{79,80}$ Ro31-9790 ${ }^{81}$ or GM6001, ${ }^{82}$ have shown a decrease the incidence and severity of EAE. Despite these encouraging preclinical results, the research was not pushed to use these broad-spectrum inhibitors in clinical trials for MS treatment, due to the beneficial role that some MMPs may have in promoting recovery of the CNS after injury. ${ }^{83}$

For many years, the interest of the scientific community was not only focused on searching for agents able to block MMPs but also on evaluating the capacity of conventional MS drugs to regulate MMP activity and expression. In this respect, numerous studies have shown that the beneficial effect of MS drugs may be in part due to their inhibitory effect on MMPs.

The early studies date back to about 20 years ago, when Rosenberg et $\mathrm{al}{ }^{61}$ showed that the steroid methylprednisolone, used to treat acute episodes of MS, reduced CSF MMP-9 levels and the number of enhancing MRI lesions in MS patients during acute exacerbations. The mechanism of inhibition of MMP-9 by steroids may be related to their known ability to block the activation of AP1, which is involved in MMP-9 gene transcription. ${ }^{84}$

In the same year, in in vitro studies on culture cells, Leppert at $a l^{85}$ and Stüve et $a l^{86}$ showed that the therapeutic efficacy of IFN $\beta$ could be due to its capacity to reduce lymphocyte trafficking through the BBB as a result of its inhibitory action on MMP-9. Afterward, numerous other studies have evidenced the IFN $\beta$ ability to modulate MMPs. In particular, several authors found a significant correlation between the CSF or serum MMP-9 levels and a decrease in active lesions during the treatment of MS patients with IFN $\beta .^{6,87,88}$ As demonstrated by an in vitro study on glial cells, the clinical efficacy of IFN $\beta$ may be due not only to its action on immune cells but also its 
ability to interfere with the capacity of activated resident CNS cells to produce MMPs. ${ }^{89}$ Other studies with the immunosuppressive drug mitoxantrone demonstrated that the inhibitory effects of this drug on the migratory ability of peripheral blood mononuclear cells are mediated by reduced levels of MMP-9.?

Also, the recent FDA-approved immunosuppressive agent fingolimod (FTY720), used for the treatment of non-IFN $\beta$ responder RR-MS patients, has shown potential in the ability to regulate MMP-9. FTY720 is a sphingosine-1-phosphate analogue able to reduce the frequency of clinical relapses and MRI-defined disease activity in MS patients. ${ }^{90}$ These effects have mainly been attributed to the ability of FTY720 to restrict immune-cell entry into the CNS via sequestration of T and B lymphocytes in secondary lymph nodes. ${ }^{91}$ Only one study has investigated the effect of this drug on MMP production. In this study, to investigate the possible pathways by which FTY720 exerts its efficacy, Foster et $\mathrm{al}^{8}$ analyzed a broad spectrum of inflammatory and immune-response genes in the CNS of EAE animals under FTY720 treatment. The authors demonstrated the ability of FTY720 to downregulate the $M M P 9$ gene and upregulate $T I M P-1$ gene, resulting in a proteolytic balance that preserved the BBB integrity. Given these findings and given the excellent ability of this lipophilic compound to pass through the $\mathrm{BBB}$, as well as its massive brain accumulation, ${ }^{92}$ it is conceivable that FTY720 may affect brain cells directly by modulating inflammatory factors, such as MMPs. In another study, Khademi et $\mathrm{al}^{9}$ analyzed the intrathecal and systemic expression of a panel of inflammatory mediators in natalizumab-treated RR-MS patients and found that MMP-9 levels were reduced in CSF from these patients.

Another approach to block the deleterious MMPs in MS is the use of CMTs, which are able to cross the BBB and possess anti-inflammatory activity. Preclinical studies in animal models of MS have shown that minocycline is able to inhibit the enzymatic activity and expression of different MMPs and to attenuate T-cell migration across a fibronectin barrier through MMP-9 inhibition. ${ }^{93,94}$ Given these encouraging results in animal models of MS, clinical trials with minocycline on RR-MS patients have been carried out. In the first pilot study, carried out by Metz et al, ${ }^{95}$ decreased gadolinium-enhancing activity in RR-MS patients treated with minocycline was shown. Subsequently, Zabad et $\mathrm{al}^{96}$ observed that serum MMP-9 levels were reduced in RR-MS patients receiving minocycline. These findings have led to the hypothesis that the effect of minocycline on the reduction in active CNS lesions might be due to an attenuation of BBB degradation as a consequence of their inhibitory effect on MMPs. Recent studies using minocycline in combination with common MS drugs have strongly encouraged their possible future application in MS treatment. ${ }^{4,97,98}$ Such a complementary therapy, thanks to the synergistic effects of the MS drugs, may be able to counteract multiple mechanisms implicated in the neuroinflammation and neurodegeneration occurring in the course of MS.

Thus far, much attention has been given to the immune response and inflammatory processes implicated in the pathogenesis of MS, whereas the role played by oxidative stress has been little considered. The CNS is particularly vulnerable to oxidative stress, due to its high oxygen consumption, weakly antioxidant systems, and the high content of biomacromolecules susceptible to oxidation. Several studies have shown that ROS play a pivotal role in both the early and the chronic stage of MS. The excessive generation of ROS may be directly or indirectly implicated in the pathogenesis of MS. ${ }^{99}$ Many studies have highlighted that ROS can activate the pathways involved in the inflammatory response, leading consequently to an increased production of neurotoxic factors, such as MMPs. ${ }^{100}$ Therefore, complementary therapies based on the use of antioxidants together with pharmacological therapy could be useful in improving disease outcome and the wellness of MS patients.

In vitro and in vivo studies on animal model of MS have indicated that antioxidants that are able to cross the BBB and reach the active inflammatory sites in the brain parenchyma have high curative potential. These antioxidants would act not only on systemic immune cells but also on brain-resident cells involved in inflammation and neurodegeneration. In an in vivo study, Marracci et $\mathrm{al}^{101}$ showed that $\alpha$-lipoic acid (LA), an antioxidant able to cross the BBB, supplied in the diet, inhibited EAE, interfering with the passage of encephalitogenic $\mathrm{T}$ cells into the spinal cord. Based on these findings, the authors concluded that the immunomodulatory effects of LA involved several mechanisms of action related to MMP-9 inhibition. In a clinical trial in MS patients, Yadav et $\mathrm{al}^{102}$ also found that LA was able to counteract increased serum levels of MMP-9. In another study, the supplementation of $\omega 3$ PUFA in patients with RR-MS significantly decreased MMP-9 levels in serum and supernatants from peripheral blood mononuclear cells. ${ }^{49}$

Recently, Riccio et al $^{12}$ investigated the influence of nutritional intervention on inflammatory status and wellness in RR-MS patients under IFN $\beta$ treatment and in untreated PP-MS patients. In particular, in this pilot study they investigated the effects of a calorie-restricted, semivegetarian diet, supplemented with vitamin D, fish oil, LA, $\omega 3$ PUFA, resveratrol and a multivitamin complex. After 6 months of this complementary treatment, although no significant 
changes in neurological signs were observed, an improvement in physical health and a decrease in MMP-9 serum levels were detected in both groups of MS patients. These results indicate that nutrition is important in MS patients, since it may contribute to reducing inflammatory status by modulating at the molecular level numerous inflammatory pathways involved in the activation of MMP-9 transcription.

\section{Conclusion}

Experimental studies have assessed that the overproduction of MMPs and failure to control their activity and expression are involved at different levels in the pathogenesis of MS. Therefore, MMP blocking at both peripheral and central levels may represent a valuable therapeutic strategy.

Here, we focused attention on the effects that specific antiMS drugs and natural compounds exert on MMPs, showing that their efficacy in MS pathology is also due to their ability to inhibit MMPs. In this scenario, therapeutic strategies aimed at blocking MMPs at both systemic and central levels would help to stop the development and the degenerative processes occurring in MS. As such, it is unlikely that current therapies, aimed mainly at blocking inflammatory cells at the systemic level, can prevent the deleterious action of MMPs within the CNS.

To overcame this gap, future research should focus on the development of drugs easily able to cross the BBB and exert their action on MMPs produced from CNS-resident cells. A first approach in this direction should be the use of combination therapies that by the association of drugs and compounds with different mechanisms of action, may exploit their synergistic action on different inflammatory pathways involved in the activation of MMPs, responsible for the progression of the disease.

A second valuable therapeutic approach may be the use of noninvasive delivery systems, based on the use of NPs, that allow drugs to cross the $\mathrm{BBB}$ and reach their targets in adequate amounts. In this respect, the use of NPs as a nonviral vector to deliver MMP-9 siRNA in brain microvascular endothelial cells should represent a useful tool for more specific targeting of MMPs. Future studies examining the impact of such novel therapeutic strategies on MS should be strongly encouraged.

\section{Disclosure}

The authors report no conflicts of interest in this work.

\section{References}

1. Lassmann H. Multiple sclerosis: lessons from molecular neuropathology. Exp Neurol. 2014;262 Pt A:2-7.

2. Rosenberg GA. Matrix metalloproteinases and neuroinflammation in multiple sclerosis. Neuroscientist. 2002;8(6):586-595.
3. Martin R, Sospedra M, Rosito M, Engelhardt B. Current multiple sclerosis treatments have improved our understanding of MS autoimmune pathogenesis. Eur J Immunol. 2016;46(9):2078-2090.

4. Chen X, Ma X, Jiang Y, Pi R, Liu Y, Ma L. The prospects of minocycline in multiple sclerosis. J Neuroimmunol. 2011;235(1-2):1-8.

5. Riccio P, Rossano R. Nutrition facts in multiple sclerosis. ASN Neuro. 2015;7(1):1759091414568185.

6. Trojano M, Avolio C, Liuzzi GM, et al. Changes of serum sICAM-1 and MMP-9 induced by $\mathrm{rIFN} \beta-1 \mathrm{~b}$ treatment in relapsing-remitting MS. Neurology. 1999;53(7):1402-1408.

7. Kopadze T, Dehmel T, Hartung HP, Stüve O, Kieseier BC. Inhibition by mitoxantrone of in vitro migration of immunocompetent cells: a possible mechanism for therapeutic efficacy in the treatment of multiple sclerosis. Arch Neurol. 2006;63(11):1572-1578.

8. Foster CA, Mechtcheriakova D, Storch MK, et al. FTY720 rescue therapy in the dark agouti rat model of experimental autoimmune encephalomyelitis: expression of central nervous system genes and reversal of blood-brain-barrier damage. Brain Pathol. 2009;19(2):254-266.

9. Khademi M, Bornsen L, Rafatnia F, et al. The effects of natalizumab on inflammatory mediators in multiple sclerosis: prospects for treatmentsensitive biomarkers. Eur J Neurol. 2009;16(4):528-536.

10. Liuzzi GM, Latronico T, Rossano R, Viggiani S, Fasano A, Riccio P. Inhibitory effect of polyunsaturated fatty acids on MMP-9 release from microglial cells: implications for complementary multiple sclerosis treatment. Neurochem Res. 2007;32(12):2184-2193.

11. Liuzzi GM, Latronico T, Branà MT, et al. Structure-dependent inhibition of gelatinases by dietary antioxidants in rat astrocytes and sera of multiple sclerosis patients. Neurochem Res. 2011;36(3):518-527.

12. Riccio P, Rossano R, Larocca M, et al. Anti-inflammatory nutritional intervention in patients with relapsing-remitting and primary-progressive multiple sclerosis: a pilot study. Exp Biol Med (Maywood). 2016;241(6):620-635.

13. Murphy G, Nagase H. Progress in matrix metalloproteinase research. Mol Aspects Med. 2008;29(5):290-308.

14. Vincenti MP, Brinckerhoff CE. Signal transduction and cell-type specific regulation of matrix metalloproteinase gene expression: can MMPs be good for you? J Cell Physiol. 2007;213(2):355-364.

15. Visse R, Nagase H. Matrix metalloproteinases and tissue inhibitors of metalloproteinases: structure, function, and biochemistry. Circ Res. 2003;92(8):827-839.

16. Baker AH, Edwards DR, Murphy G. Metalloproteinase inhibitors: biological actions and therapeutic opportunities. JCell Sci. 2002;115(Pt 19): 3719-3727.

17. Yamamoto K, Murphy G, Troeberg L. Extracellular regulation of metalloproteinases. Matrix Biol. 2015;44-46:255-263.

18. Brew K, Dinakarpandian D, Nagase H. Tissue inhibitors of metalloproteinases: evolution, structure and function. Biochim Biophys Acta. 2000;1477(1-2):267-283.

19. Amoura A, Slocombeb PM, Websterb A, et al. TNF- $\alpha$ converting enzyme (TACE) is inhibited by TIMP-3. FEBS Lett. 1998;435(1): $39-44$.

20. Hu J, Van den Steen PE, Sang QX, Opdenakker G. Matrix metalloproteinase inhibitors as therapy for inflammatory and vascular diseases. Nat Rev Drug Discov. 2007;6(6):480-498.

21. Vihinen P, Ala-aho R, Kähäri VM. Matrix metalloproteinases as therapeutic targets in cancer. Curr Cancer Drug Targets. 2005; 5(3):203-220.

22. Overall CM, Kleifeld O. Tumour microenvironment - opinion: validating matrix metalloproteinases as drug targets and anti-targets for cancer therapy. Nat Rev Cancer. 2006;6(3):227-239.

23. Skuljec J, Gudi V, Ulrich R, et al. Matrix metalloproteinases and their tissue inhibitors in cuprizone-induced demyelination and remyelination of brain white and gray matter. J Neuropathol Exp Neurol. 2011;70(9):758-769.

24. Johnson AR, Pavlovsky AG, Ortwine DF, et al. Discovery and characterization of a novel inhibitor of matrix metalloprotease- 13 that reduces cartilage damage in vivo without joint fibroplasia side effects. $J$ Biol Chem. 2007;282(38):27781-27791. 
25. Jacobsen JA, Jourden JL, Miller MT, Cohen SM. To bind zinc or not to bind zinc: an examination of innovative approaches to improved metalloproteinase inhibition. Biochim Biophys Acta. 2010;1803(1):72-94.

26. Bonfil RD, Sabbota A, Nabha $\mathrm{S}$, et al. Inhibition of human prostate cancer growth, osteolysis and angiogenesis in a bone metastasis model by a novel mechanism-based selective gelatinase inhibitor. Int J Cancer. 2006;118(11):2721-2726.

27. Cui J, Chen S, Zhang C, et al. Inhibition of MMP-9 by a selective gelatinase inhibitor protects neurovasculature from embolic focal cerebral ischemia. Mol Neurodegener. 2012;7:21.

28. Hadass O, Tomlinson BN, Gooyit M, et al. Selective inhibition of matrix metalloproteinase-9 attenuates secondary damage resulting from severe traumatic brain injury. PLoS One. 2013;8(10):e76904.

29. Koivunen E, Arap W, Valtanen H, et al. Tumor targeting with a selective gelatinase inhibitor. Nat Biotechnol. 1999;17(8):768-774.

30. Garrido-Mesa N, Zarzuelo A, Gálvez J. Minocycline: far beyond an antibiotic. Br J Pharmacol. 2013;169(2):337-352.

31. Kim HS, Suh YH. Minocycline and neurodegenerative diseases. Behav Brain Res. 2009;196(2):168-179.

32. Maier K, Merkler D, Gerber J, et al. Multiple neuroprotective mechanisms of minocycline in autoimmune CNS inflammation. Neurobiol Dis. 2007;25(3):514-525.

33. Paemen L, Martens E, Norga K, et al. The gelatinase inhibitory activity of tetracyclines and chemically modified tetracycline analogues as measured by a novel microtiter assay for inhibitors. Biochem Pharmacol. 1996;52(1):105-111.

34. Martens E, Leyssen A, Van Aelst I, et al. A monoclonal antibody inhibits gelatinase B/MMP-9 by selective binding to part of the catalytic domain and not to the fibronectin or zinc binding domains. Biochim Biophys Acta. 2007;1770(2):178-186.

35. Fields GB. New strategies for targeting matrix metalloproteinases. Matrix Biol. 2015;44-46:239-246.

36. Bonoiu A, Mahajan SD, Ye L, et al. MMP-9 gene silencing by a quantum dot-siRNA nanoplex delivery to maintain the integrity of the blood brain barrier. Brain Res. 2009;1282:142-155.

37. Mahajan SD, Aalinkeel R, Reynolds JL, et al. Suppression of MMP-9 expression in brain microvascular endothelial cells (BMVEC) using a gold nanorod (GNR)-siRNA nanoplex. Immunol Invest. 2012;41(4):337-355.

38. Latronico T, Branà MT, Merra E, et al. Impact of manganese neurotoxicity on MMP-9 production and superoxide dismutase activity in rat primary astrocytes: effect of resveratrol and therapeutical implications for the treatment of CNS diseases. Toxicol Sci. 2013;135(1):218-228.

39. Saragusti AC, Ortega MG, Cabrera JL, Estrin DA, Marti MA, Chiabrando GA. Inhibitory effect of quercetin on matrix metalloproteinase 9 activity molecular mechanism and structure-activity relationship of the flavonoid-enzyme interaction. Eur J Pharmacol. 2010;644(1-3):138-145.

40. Sartor L, Pezzato E, Dell'Aica I, Caniato R, Biggin S, Garbisa S. Inhibition of matrix-proteases by polyphenols: chemical insights for anti-inflammatory and anti-invasion drug design. Biochem Pharmacol. 2002;64(2):229-237.

41. Sternberg Z, Chadha K, Lieberman A, et al. Quercetin and interferon- $\beta$ modulate immune response(s) in peripheral blood mononuclear cells isolated from multiple sclerosis patients. J Neuroimmunol. 2008;205(1-2):142-147.

42. Hsieh HL, Wang HH, Wu WB, Chu PJ, Yang CM. Transforming growth factor- $\beta 1$ induces matrix metalloproteinase- 9 and cell migration in astrocytes: roles of ROS-dependent ERK- and JNK-NF- $\mathrm{KB}$ pathways J Neuroinflammation. 2010;7:88.

43. Wang K, Wan YJ. Nuclear receptors and inflammatory diseases. Exp Biol Med (Maywood). 2008;233(5):496-506.

44. Simopoulos AP. Omega-3 fatty acids in inflammation and autoimmune diseases. J Am Coll Nutr. 2002;21(6):495-505.

45. Yates CM, Calder PC, Rainger GE. Pharmacology and therapeutics of omega-3 polyunsaturated fatty acids in chronic inflammatory disease. Pharmacol Ther. 2014;141(3):272-282.
46. Calder PC. Marine omega-3 fatty acids and inflammatory processes: effects, mechanisms and clinical relevance. Biochim Biophys Acta. 2015;1851(4):469-484.

47. Nestel P, Clifton P, Colquhoun D, et al. Indications for omega-3 long chain polyunsaturated fatty acid in the prevention and treatment of cardiovascular disease. Heart Lung Circ. 2015;24(8):769-779.

48. Dyall SC, Michael-Titus AT. Neurological benefits of omega-3 fatty acids. Neuromolecular Med. 2008;10(4):219-235.

49. Shinto L, Marracci G, Baldauf-Wagner S, et al. Omega-3 fatty acid supplementation decreases matrix metalloproteinase-9 production in relapsing-remitting multiple sclerosis. Prostaglandins Leukot Essent Fatty Acids. 2009;80(2-3):131-136.

50. Zhao Y, Chen LH. Eicosapentaenoic acid prevents lipopolysaccharidestimulated DNA binding of activator protein-1 and c-Jun N-terminal kinase activity. J Nutr Biochem. 2005;16(2):78-84.

51. Zhang C, Kim SK. Matrix metalloproteinase inhibitors (MMPIs) from marine natural products: the current situation and future prospects. Mar Drugs. 2009;7(2):71-84.

52. Thomas NV, Kim SK. Metalloproteinase inhibitors: status and scope from marine organisms. Biochem Res Int. 2010;2010:845975.

53. Di Bari G, Gentile E, Latronico T, et al. Inhibitory effect of aqueous extracts from marine sponges on the activity and expression of gelatinases A (MMP-2) and B (MMP-9) in rat astrocyte cultures. PLoS One. 2015;10(6):e0129322.

54. Compston A, Coles A. Multiple sclerosis. Lancet. 2008;372(9648): 1502-1517.

55. Oksenberg JR, Baranzini SE. Multiple sclerosis genetics: is the glass half full, or half empty? Nat Rev Neurol. 2010;6(8):429-437.

56. Lauer K. Environmental risk factors in multiple sclerosis. Expert Rev Neurother. 2010;10(3):421-440.

57. Perron H, Lang A. The human endogenous retrovirus link between genes and environment in multiple sclerosis and in multifactorial diseases associating neuroinflammation. Clin Rev Allergy Immunol. 2010;39(1):51-61.

58. McFarland HF, Martin R. Multiple sclerosis: a complicated picture of autoimmunity. Nat Immunol. 2007;8(9)913-919.

59. Zindler E, Zipp F. Neuronal injury in chronic CNS inflammation. Best Pract Res Clin Anaesthesiol. 2010;24(4):551-562.

60. Trapp BD. Pathogenesis of multiple sclerosis: the eyes only see what the mind is prepared to comprehend. Ann Neurol. 2004;55(4):455-457.

61. Rosenberg GA, Dencoff JE, Correa N Jr, Reiners M, Ford CC. Effect of steroids on CSF matrix metalloproteinases in multiple sclerosis: relation to blood-brain barrier injury. Neurology. 1996;46(6):1626-1632.

62. Leppert D, Waubant E, Galardy R, Bunnett NW, Hauser SL. T cell gelatinases mediate basement membrane transmigration in vitro. J Immunol. 1995;154(9):4379-4389.

63. Chandler S, Miller KM, Clements JM, et al. Matrix metalloproteinases, tumor necrosis factor and multiple sclerosis: an overview. J Neuroimmunol. 1997;72(2):155-161.

64. Cossins JA, Clements JM, Ford J, et al. Enhanced expression of MMP-7 and MMP-9 in demyelinating multiple sclerosis lesions. Acta Neuropathol. 1997;94(6):590-598.

65. Diaz-Sanchez M, Williams K, DeLuca GC, Esiri MM. Protein coexpression with axonal injury in multiple sclerosis plaques. Acta Neuropathol. 2006;111(4):289-299.

66. Cuzner ML, Gveric D, Strand C, et al. The expression of tissue-type plasminogen activator, matrix metalloproteases and endogenous inhibitors in the central nervous system in multiple sclerosis: comparison of stages in lesion evolution. J Neuropathol Exp Neurol. 1996;55(12):1194-1204.

67. Kouwenhoven M, Ozenci V, Gomes A, et al. Multiple sclerosis: elevated expression of matrix metalloproteinases in blood monocytes. J Autoimmun. 2001;16(4):463-470.

68. Lindberg RL, De Groot CJ, Montagne L, et al. The expression profile of matrix metalloproteinases (MMPs) and their inhibitors (TIMPs) in lesions and normal appearing white matter of multiple sclerosis. Brain. 2001;124(Pt 9):1743-1753. 
69. Vos CM, van Haastert ES, de Groot CJ, van der Valk P, de Vries HE. Matrix metalloproteinase-12 is expressed in phagocytotic macrophages in active multiple sclerosis lesions. J Neuroimmunol. 2003;138(1-2):106-114.

70. Bar-Or A, Nuttall RK, Duddy M,et al. Analyses of all matrix metalloproteinase members in leukocytes emphasize monocytes as major inflammatory mediators in multiple sclerosis. Brain. 2003;126(Pt 12): 2738-2749.

71. Maeda A, Sobel RA. Matrix metalloproteinases in the normal human central nervous system, microglial nodules, and multiple sclerosis lesions. J Neuropathol Exp Neurol. 1996;55(3):300-309.

72. Gijbels K, Masure S, Carton H, Opdenakker G. Gelatinase in the cerebrospinal fluid of patients with multiple sclerosis and other inflammatory neurological disorders. J Neuroimmunol. 1992;41(1):29-34.

73. Liuzzi GM, Trojano M, Fanelli M, et al. Intrathecal synthesis of matrix metalloproteinase-9 in patients with multiple sclerosis: implication for pathogenesis. Mult Scler. 2002;8(3):222-228.

74. Avolio C, Ruggieri M, Giuliani F, et al. Serum MMP-2 and MMP-9 are elevated in different multiple sclerosis subtypes. J Neuroimmunol. 2003;136(1-2):46-53.

75. Benesová Y, Vasku A, Novotná H, et al. Matrix metalloproteinase-9 and matrix metalloproteinase-2 as biomarkers of various courses in multiple sclerosis. Mult Scler. 2009;15(3):316-22.

76. Proost P, Van Damme J, Opdenakker G. Leukocyte gelatinase B cleavage releases encephalitogens from human myelin basic protein. Biochem Biophys Res Commun. 1993;192(3):1175-1181.

77. Chandler S, Coates R, Gearing A, Lury J, Wells G, Bone E. Matrix metalloproteinases degrade myelin basic protein. Neurosci Lett. 1995;201(3):223-226.

78. Gearing AJ, Beckett P, Cristodoulou M, et al. Processing of tumor necrosis factor- $\alpha$ precursor by metalloproteinases. Nature. 1994; 370(6490):555-557.

79. Clements JM, Cossins JA, Wells GM, et al. Matrix metalloproteinase expression during experimental autoimmune encephalomyelitis and effects of a combined matrix metalloproteinase and tumour necrosis factor- $\alpha$ inhibitor. $J$ Neuroimmunol. 1997;74(1-2):85-94.

80. Liedtke W, Cannella B, Mazzaccaro RJ, et al. Effective treatment of models of multiple sclerosis by matrix metalloproteinase inhibitors. Ann Neurol. 1998;44(1):35-46.

81. Hewson AK, Smith T, Leonard JP, Cuzner ML. Suppression of experimental allergic encephalomyelitis in the Lewis rat by the matrix metalloproteinase inhibitor Ro31-9790. Inflamm Res. 1995;44(8):345-349.

82. Gijbels K, Galardy RE, Steinman L. Reversal of experimental autoimmune encephalomyelitis with a hydroxamate inhibitor of matrix metalloproteases. J Clin Invest. 1994;94(6):2177-2182.

83. Yong VW. Metalloproteinases: mediators of pathology and regeneration in the CNS. Nat Rev Neurosci 2005;6(12):931-944.

84. Jonat C, Rahmsdorf HJ, Park KK, et al. Antitumor promotion and antiinflammation: down-modulation of AP-1 (Fos/Jun) activity by glucocorticoid hormone. Cell. 1990;62(6):1189-1204.

85. Leppert D, Waubant E, Burk MR, Oksenberg JR, Hauser SL. Interferon $\beta-1 \mathrm{~b}$ inhibits gelatinase secretion and in vitro migration of human $\mathrm{T}$ cells: a possible mechanism for treatment efficacy in multiple sclerosis. Ann Neurol. 1996;40(6):846-852.

86. Stüve O, Dooley NP, Uhm JH, et al. Interferon $\beta-1 \mathrm{~b}$ decreases the migration of $\mathrm{T}$ lymphocytes in vitro: effects on matrix metalloproteinase-9. Ann Neurol. 1996;40(6):853-863.

87. Avolio C, Filippi M, Tortorella C, et al. Serum MMP-9/TIMP-1 and MMP-2/TIMP-2 ratios in multiple sclerosis: relationships with different magnetic resonance imaging measures of disease activity during IFN- $\beta$-1a treatment. Mult Scler. 2005;11(4):441-446.
88. Boz C, Ozmenoglu M, Velioglu S, et al. Matrix metalloproteinase-9 (MMP-9) and tissue inhibitor of matrix metalloproteinase (TIMP-1) in patients with relapsing-remitting multiple sclerosis treated with interferon beta. Clin Neurol Neurosurg. 2006;108(2): 124-128.

89. Liuzzi GM, Latronico T, Fasano A, Carlone G, Riccio P. Interferon- $\beta$ inhibits the expression of metalloproteinases in rat glial cell cultures: implications for multiple sclerosis pathogenesis and treatment. Mult Scler. 2004;10(3):290-297.

90. Khatri BO. Fingolimod in the treatment of relapsing-remitting multiple sclerosis: long-term experience and an update on the clinical evidence. Ther Adv Neurol Disord. 2016;9(2):130-147.

91. Gräler MH, Goetzl EJ. The immunosuppressant FTY720 down-regulates sphingosine 1-phosphate G-protein-coupled receptors. FASEB J. 2004;18(3):551-553.

92. Miron VE, Schubart A, Antel JP. Central nervous system-directed effects of FTY720 (fingolimod). J Neurol Sci. 2008;274(1-2):13-17.

93. Brundula V, Rewcastle NB, Metz LM, Bernard CC, Yong VW. Targeting leukocyte MMPs and transmigration: minocycline as a potential therapy for multiple sclerosis. Brain. 2002;125(Pt 6):1297-1308.

94. Popovic N, Schubart A, Goetz BD, Zhang SC, Linington C, Duncan ID. Inhibition of autoimmune encephalomyelitis by a tetracycline. Ann Neurol. 2002;51(2):215-223.

95. Metz LM, Zhang Y, Yeung M, et al. Minocycline reduces gadoliniumenhancing magnetic resonance imaging lesions in multiple sclerosis. Ann Neurol. 2004;55(5):756.

96. Zabad RK, Metz LM, Todoruk TR, et al. The clinical response to minocycline in multiple sclerosis is accompanied by beneficial immune changes: a pilot study. Mult Scler. 2007;13(4):517-526.

97. Minagar A, Alexander JS, Schwendimann RN, et al. Combination therapy with interferon $\beta$-1a and doxycycline in multiple sclerosis: an open-label trial. Arch Neurol. 2008;65(2):199-204.

98. Ruggieri M, Pica C, Lia A, et al. Combination treatment of glatiramer acetate and minocycline affects phenotype expression of blood monocyte-derived dendritic cells in multiple sclerosis patients. J Neuroimmunol. 2008;197(2):140-146.

99. van Horssen J, Witte ME, Schreibelt G, de Vries HE. Radical changes in multiple sclerosis pathogenesis. Biochim Biophys Acta. 2011;1812(2):141-150.

100. Hsieh HL, Yang CM. Role of redox signaling in neuroinflammation and neurodegenerative diseases. Biomed Res Int. 2013;2013:484613.

101. Marracci GH, Jones RE, McKeon GP, Bourdette DN. Alpha lipoic acid inhibits $\mathrm{T}$ cell migration into the spinal cord and suppresses and treats experimental autoimmune encephalomyelitis. J Neuroimmunol. 2002;131(1-2):104-114.

102. Yadav V, Marracci G, Lovera J, et al. Lipoic acid in multiple sclerosis: a pilot study. Mult Scler. 2005;11(2):159-165.

103. Montalban X, Hammer B, Rammohan K, et al. Efficacy and safety of ocrelizumab in primary progressive multiple sclerosis: results of the placebo-controlled, double-blind, phase III ORATORIO study. 2015. Available from: http://onlinelibrary.ectrims-congress. eu/ectrims/2015/31st/116701/xavier.montalban.efficacy.and.safety. of.ocrelizumab.in.primary.progressive.html? $\mathrm{f}=\mathrm{m} 3$. Accessed November 24, 2016.

104. Giuliani F, Fu SA, Metz LM, Yong VW. Effective combination of minocycline and interferon- $\beta$ in a model of multiple sclerosis. J Neuroimmunol. 2005;165(1-2):83-91.

105. Giuliani F, Metz LM, Wilson T, Fan Y, Bar-Or A, Yong VW. Additive effect of the combination of glatiramer acetate and minocycline in a model of MS. J Neuroimmunol. 2005;158(1-2):213-221. 
Metalloproteinases In Medicine is an international, peer reviewed, open access journal that aims to provide a platform for the discussion and dissemination of knowledge about the role that metalloproteinases - such as matrix metalloproteinases (MMP), ADAMs, ADAMTSs, and astacins, as well as their inhibitors - play in diseases.

\section{Dovepress}

The manuscript management system is completely online and includes a very quick and fair peer review system, which is all easy to use. Visit http://www.dovepress.com/testimonials.php to read real quotes from published authors.

Submit your manuscript here: https://www.dovepress.com/metalloproteinases-in-medicine-journal 